\title{
THE CENTER OF AN ORDER WITH FINITE GLOBAL DIMENSION
}

\author{
BY \\ MARK RAMRAS
}

\begin{abstract}
Let $\Lambda$ be a quasi-local ring of global dimension $n<\infty$. Assume that its center $R$ is a noetherian domain, that $\Lambda$ is finitely generated torsionfree as an $R$-module, and that $R$ is an $R$-direct summand of $\Lambda$. Then $R$ is integrally closed in its quotient field $K$ and Macauley of dimension $n$. Furthermore, when $n=2, \Lambda$ is a maximal $R$-order in the central simple $K$-algebra $\Lambda \otimes_{R} K$. This extends an earlier result of the author, in which $R$ was assumed to have global dimension 2. Examples are given to show that in the above situation $R$ can have infinite global dimension.
\end{abstract}

Introduction. Let $R$ be a commutative noetherian domain with quotient field $K$, and let $\Lambda$ be a central $R$-algebra which is finitely generated and torsionfree as an $R$-module. Assume further that $R$ is an $R$-direct summand of $\Lambda$. Then if the global dimension of $\Lambda$ is $n$ and $\Lambda$ is quasi-local, $R$ is integrally closed in $K$ and Macauley of dimension $n$. Harada [7] had proved that when $n=1, R$ is a Dedekind domain, without assuming that $\Lambda$ is quasi-local, or that $R$ is an $R$-direct summand of $\Lambda$.

When $n=2$ we can say more. Then $\Lambda$ is $R$-reflexive and, in fact, is a maximal $R$-order in the central simple $K$-algebra $\Sigma=\Lambda \otimes_{R} K$. This extends an earlier result of the author [13] in which $R$ was assumed to be regular local of dimension 2.

Using skew power series rings we give a general method for constructing examples of local orders with finite global dimension whose centers have infinite global dimension. By means of these examples we show that the abovementioned result is indeed an extension of the earlier one. We conjecture that, in general, these skew power series orders are maximal.

0. Notation and conventions. In this paper all rings have 1 and all modules are unital. $R$ will always denote a commutative noetherian local domain with quotient field $K$. $\Lambda$ will denote a central $R$-algebra, finitely generated and torsion-free as an $R$-module. Gl. dim. stands for global dimension, while 1.p.d. and

Received by the editors July 24, 1974.

AMS (MOS) subject classifications (1970). Primary 16A18, 16A60; Secondary $13 \mathrm{H} 10$.

Key words and phrases. Maximal order, global dimension, center, Macauley ring, integrally closed, skew power series ring. 
and 1.i.d. denote left projective dimension and left injective dimension, respectively.

1. Preliminary results. Let $(A, \mathfrak{m})$ be a commutative noetherian local ring and $\Gamma$ any $A$-algebra which is finitely generated as an $A$-module. For a multiplicatively closed subset $S$ of $A-\{0\}, A_{S}$ will denote the localization of $A$ at $S$, and for any $A$-module $M, M_{S}=A_{S} \otimes_{A} M$. Furthermore, if $\mathfrak{p}$ is a prime ideal of $A$, we will follow the usual convention and write $M_{p}$ instead of $M_{A-p}$.

LEMMA 1.1. Let $S$ be a multiplicatively closed subset of $A-\{0\}$ such that $\mathfrak{m} \cap S \neq \varnothing$. Assume that $\Gamma_{S} \neq 0$. If 1.i.d. $\Gamma \Gamma<\infty$, then 1.i.d. $\Gamma_{S} \Gamma_{S}<1$. i.d. ${ }_{\Gamma} \Gamma$.

Proof. The proof of [13, Proposition 3.1] works if the hypothesis about the regularity of elements of $S$ on $\Gamma$ is replaced by the hypothesis that $\Gamma_{S} \neq$ 0 .

Corollary 1.2. If $\Gamma \supset A$ then 1.i.d. $\Gamma$ $\Gamma \geqslant \operatorname{Krull} \operatorname{dim} . A$.

Proof. This follows from Lemma 1.1 by an easy induction on $n=$ Krull $\operatorname{dim} . A$.

Corollary 1.3. Let $A$ be a Macauley ring. Suppose that $\Gamma \supset A$ and 1.i.d. $\Gamma=\operatorname{Krull} \operatorname{dim} A$. Then for any $\mathfrak{p} \in \operatorname{Spec} A, 1$.i.d. $\Gamma_{\mathfrak{p}} \Gamma_{\mathfrak{p}}=\operatorname{Krull} \operatorname{dim} . A_{\mathfrak{p}}$. In particular, if $\mathrm{gl} . \operatorname{dim} . \Gamma=\mathrm{Krull} \operatorname{dim} . A$, then $\mathrm{gl} . \operatorname{dim} . \Gamma_{\mathfrak{p}}=\mathrm{Krull} \operatorname{dim} . A_{\mathfrak{p}}$.

Proof. Since $A$ is Macauley, there is a saturated chain of prime ideals $\mathbf{m}=$ $\mathfrak{p}_{0} \supsetneqq \mathfrak{p}_{1} \supsetneqq \cdots \supsetneqq \mathfrak{p}_{t}=\mathfrak{p}$ such that $t+\mathrm{Krull} \operatorname{dim} . A_{\mathfrak{p}}=\mathrm{Krull} \operatorname{dim} . A$. By Lemma 1.1,

$$
\text { 1.i.d. } \Gamma_{\Gamma} \Gamma_{\mathrm{h}} \leqslant\left(1 . \text { i.d. }{ }_{\Gamma} \Gamma\right)-t=(\operatorname{Krull} \operatorname{dim} . A)-t=\operatorname{Krull} \operatorname{dim} . A_{\curvearrowright} \text {. }
$$

But by the preceding corollary, 1.i.d. $\Gamma_{p} \Gamma_{p} \geqslant \operatorname{Krull} \operatorname{dim} . A_{p}$, so we have the desired equality. The second statement follows from the fact that the global dimension, when finite, equals the injective dimension, and the fact that the finiteness is preserved under localization.

Corollary 1.4. Suppose $A$ is a Macauley domain with quotient field $F$, and $\Gamma$ is a central A-algebra. If $\mathrm{gl}$. $\operatorname{dim} . \Gamma=\mathrm{Krull} \operatorname{dim} . A$, then $\Gamma \otimes_{A} F$ is a central simple F-algebra.

Proof. By Corollary $1.3, \mathrm{gl}$. dim. $\Gamma \otimes_{A} F=0$, so that $\Gamma \otimes_{A} F$ is semisimple. Its center is $F$ (since $\Gamma$ is $A$-central) and since $F$ is a field, the semisimple 
ring $\Gamma \otimes_{A} F$ must, in fact, be simple (otherwise $F$ would be a direct product of the centers of the simple components of $\Gamma \otimes_{A} F$ ). Hence $\Gamma \otimes_{A} F$ is a central simple $F$-algebra.

Recall that for an $A$-module $M$, depth $A$ (or $\operatorname{codh}_{A} M$ in [2]) is the length of the longest $M$-regular sequence contained in the maximal ideal (or more generally, for nonlocal rings, the Jacobson radical) of $A$.

$\Gamma$ is called quasi-local if $\Gamma / J(\Gamma)$ is a simple artin ring, where $J(\Gamma)$ is the Jacobson radical of $\Gamma$.

Proposition 1.5. Suppose $\Gamma \supset A, \Gamma$ is quasi-local, and 1.i.d. $\Gamma=n<\infty$. If $\operatorname{depth}_{A} \Gamma \leqslant \operatorname{depth}_{A} A$ then $A$ is a Macauley ring of dimension $n$. (In particular, this inequality is satisfied if $A$ is an A-direct summand of $\Gamma$, or if p.d. $\boldsymbol{A} \Gamma<\infty$.)

Proof. Since $\Gamma$ is quasi-local, it follows from [13, Corollary 2.15] that $n=1$.i.d. $\Gamma=\operatorname{depth}_{A} \Gamma$. By Corollary 1.2, Krull dim. $A \leqslant n$. Hence Krull dim. $A \leqslant \operatorname{depth}_{A} A$. Thus Krull dim. $A=\operatorname{depth}_{A} A$ and $A$ is Macauley of dimension $n$.

We turn now to the domain $R$ and the central $R$-algebra $\Lambda$.

THEOREM 1.6. Assume that $R$ is an $R$-direct summand of $\Lambda$. Suppose that $\Lambda$ is quasi-local and $\mathrm{gl} . \operatorname{dim} . \Lambda=n<\infty$. Then $R$ is integrally closed and Macauley of dimension $n$.

Proof. The preceding proposition shows that $R$ is Macauley of dimension $n$. Hence, in particular, every principal ideal of $R$ is unmixed. Therefore, according to [12,Theorem 12.9, p. 241$]$, to show that $R$ is integrally closed, it suffices to show that for each height one prime $\mathfrak{p}, R_{\mathfrak{p}}$ is integrally closed (or equivalently, a discrete valuation ring). Since $\mathrm{gl} . \operatorname{dim} . \Lambda=\operatorname{Krull} \operatorname{dim} . R$, and $R$ is Macauley, if height $\mathfrak{p}=1$, then by Corollary 1.3 , gl. $\operatorname{dim} . \Lambda_{\mathfrak{p}}=\operatorname{Krull} \operatorname{dim} . R_{\mathfrak{p}}=1$. Thus $\Lambda_{\mathfrak{p}}$ is a hereditary order in the $K$-algebra $\Lambda \otimes_{R} K$, which by Corollary 1.4 is central simple. By a result of Harada [7, Theorem 2.6] the center of $\Lambda_{p}, R_{p}$, is a discrete valuation ring. Thus $R$ is integrally closed.

2. The case $n=2$. In this section, we obtain sharper results when gl. $\operatorname{dim} . \Lambda=2$. We begin with a general observation.

Proposition 2.1. Suppose $\Omega \subset \Gamma$ are orders in the same quotient ring. If $\Gamma$ is a finitely generated projective generator in the category of right $\Omega$-modules, then $\Omega=\Gamma$.

Proof. View $\Gamma$ as a $\Gamma-\Omega$ bimodule. By Morita duality, $\Omega=\operatorname{End}_{\Delta}(\Gamma)$, 
where $\Delta=\operatorname{End}_{\Omega}(\Gamma)$. For $\gamma \in \Gamma$, let $L_{\gamma}: \Gamma \rightarrow \Gamma$ denote left multiplication by $\gamma$. Then $L_{\gamma} \in \Delta$, and $\gamma \sim L_{\gamma}$ defines a ring homomorphism $\Gamma \rightarrow \Delta$ which is clearly one-to-one. On the other hand, if $\sigma \in \Delta$, then $\sigma$ extends to a right $\Sigma$-endomorphism of $\Sigma$, where $\Sigma$ is the common quotient ring of $\Gamma$ and $\Omega$. This endomorphism is just left multiplication by some element $\alpha \in \Sigma$. Hence $\sigma$ itself is left multiplication by $\alpha$, and $\alpha \Gamma=\sigma(\Gamma) \subset \Gamma$, so $\alpha \in \Gamma$. Hence $\sigma=L_{\alpha}$, and the map $\gamma \sim L_{\gamma}$ is an isomorphism of $\Gamma$ onto $\Delta$. Hence $\Omega=\operatorname{End}_{\Delta}(\Gamma)=\operatorname{End}_{\Gamma}(\Gamma)$. $\Gamma$ is now being viewed as a right $\Gamma$-module, and so $\operatorname{End}_{\Gamma}(\Gamma)=\Gamma$. Hence $\Omega=\Gamma$.

THEOREM 2.2. Suppose $\Lambda$ is quasi-local and gl. $\operatorname{dim} . \Lambda=2$. Let $R$ be the center of $\Lambda$ Assume that $R$ is a domain with quotient field $K$ and $\Lambda$ is a finitely generated torsion-free $R$-module. Assume further that $R$ is an $R$-direct summand of $\Lambda$. Let $\Sigma=\Lambda \otimes_{R} K$. Then $\Lambda$ is $R$-reflexive and a maximal $R$-order in the central simple K-algebra $\Sigma$.

Proof. Note that $R$ is local since $\Lambda$ is quasi-local, $\Lambda \supset R$, and $\Lambda$ is a finite $R$-module. By Theorem 1.6, $R$ is integrally closed in $K$ and Macauley of dimension 2. Let $\Gamma$ be a maximal $R$-order in $\Sigma$ which contains $\Lambda$. Then $\Gamma$ is $R$-reflexive [3, Theorem 1.5]. Hence depth $R \geqslant 2$ [1, Lemma 2]. If $x, y$ is a $\Gamma$-regular sequence contained in $R$, then

$$
\text { p.d. } \Gamma /(x, y) \Gamma=2+\text { p.d. }{ }_{\Lambda} \Gamma \leqslant \text { gl. } \operatorname{dim} . \Lambda=2 \text {. }
$$

Hence p.d. $\Lambda=0$, i.e. $\Gamma$ is $\Lambda$-projective. Since $\Lambda$ is quasi-local, every nonzero projective $\Lambda$-module is a projective generator. By Proposition $2.1, \Lambda=\Gamma$. Hence $\Lambda$ is $R$-reflexive and a maximal $R$-order in $\Sigma$.

We can strengthen the preceding theorem when $R$ is complete (or equivalently, when $\Lambda$ is complete in the $J(\Lambda)$-adic topology).

THEOREM 2.3. Suppose gl. $\operatorname{dim} . \Lambda=2$ and $\Lambda$ is quasi-local. Assume that its center, $R$, is an $R$-direct summand of $\Lambda$. Suppose the local domain $R$ is complete. If $\Omega$ is any maximal $R$-order in $\Sigma=\Lambda \otimes_{R} K$, then $\Omega=t \Lambda t^{-1}$ for some unit $t \in \Sigma$.

Proof. Since $R$ is a complete local domain, it contains a complete regular local ring $S$ such that $R$ is a finite $S$-module [12, Corollary 31.6, p. 109]. Furthermore, gl. $\operatorname{dim} . S=2$ by [13, Corollary 2.17]. Let $L$ be the quotient field of $S$. Then $K$ is finite dimensional over $L$, and since $L \subset R L \subset K, R L$ is a field. Since $R \subset R L, R L=K$, the quotient field of $R$. Thus if $\Gamma$ is any $R$-order in $\Sigma$, $\Sigma=\Gamma K=\Gamma R L=\Gamma L$, and hence $\Gamma \otimes_{S} L=\Sigma$. Since $\Gamma$ is a finite $R$-module, it is also finite as an $S$-module. Thus $\Gamma$ is an $S$-order in $\Sigma$. Now consider the maxi- 
mal $R$-order $\Omega$. We claim that it is also maximal as an $S$-order in $\Sigma$. For if $\Delta$ is any $S$-order in $\Sigma$, then $\Delta R$ is an $R$-order in $\Sigma$. Thus if $\Omega \subset \Delta$, then since $\Delta \subset \Delta R$, we have $\Omega \subset \Delta R$ and by the $R$-maximality of $\Omega, \Omega=\Delta R$. Hence $\Omega=\Delta$, and $\Omega$ is indeed $S$-maximal. We now appeal to [13, Theorem 5.4], observing that nowhere in its proof did we use the fact that the regular local ring of dimension 2 was actually equal to the center of $\Lambda$, but only that $\Lambda$ was finitely generated as a module over this ring.

In the next section we shall give an example to show that the hypotheses of Theorem 2.3 do not imply that $R$ is regular local, and thus the theorem is a generalization of [13, Theorem 5.4].

THEOREM 2.4. Let $R$ be a noetherian integrally closed local domain, and $\Lambda$ an $R$-reflexive $R$-order in the central simple $K$-algebra $\Sigma$. Assume that $R$ is an $R$-direct summand of $\Lambda$. Suppose that for some nonzero, nonunit $x \in R,(x)$ is

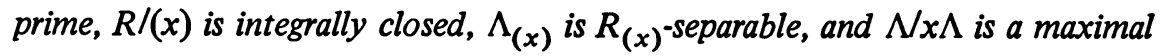
$R /(x)$-order in $\Lambda_{(x)} / x \Lambda_{(x)}$. Then $\Lambda$ is maximal.

Proof. The proof of [14, Theorem 3.1] works here, except that where [13, Theorem 5.4] was invoked, we now use Theorem 2.2 instead.

COROllaRy 2.5. Let $R$ be integrally closed in $K$, and $\Lambda$ a maximal $R$-order in the central simple K-algebra $\Sigma$. Assume that $R$ is an R-direct summand of $\Lambda$. Then $\Lambda[[X]]=\Lambda \otimes_{R} R[[X]]$ is a maximal $R[[X]]$-order in $\Sigma \otimes_{K} L$, where $L$ is the quotient field of $R[[X]]$.

Proof. $R[[X]]$ is integrally closed in $L$ and is an $R[[X]]$-direct summand of $\Lambda[[X]]$. Since $\Lambda$ is a maximal $R$-order, it is $R$-reflexive. $\Lambda[[X]]_{(X)} / X \Lambda[[X]]_{(X)} \simeq$ $\Lambda \otimes_{R} K=\Sigma$. Therefore $\Lambda[[X]]_{(X)}$ is $R[[X]]_{(X)}$-separable. Theorem 2.4 now applies.

3. Skew power series rings. We begin this section with some general observations abcut the ring of invariants $A^{G}$ of a commutative ring $A$ under the action of a finite group of automorphisms $G$.

Lemma 3.1. Suppose $o(G)$, the order of $G$, is invertible in $A$. Then $A^{G}$ is an $A^{G}$-direct summand of $A$.

Proof. Let $n=o(G)$. Define $f: A \rightarrow A^{G}$ by $f(a)=n^{-1} \Sigma_{\sigma \in G} \sigma(a)$. Clearly $f$ is $A^{G}$-linear and the restriction of $f$ to $A^{G}$ is the identity map.

CoRollary 3.2 (Hochster AND EAgON). Suppose o(G) is invertible in $A$. If $A$ is Macauley, then so is $A^{G}$. 
Proof. See [8, Proposition 13].

LEMMA 3.3. Let $R$ be an integrally closed noetherian domain, and let $T \supset R$ be a commutative ring which is a finite R-module. Suppose $T$ is Macauley. Then $T$ is $R$-reflexive.

Proof. It suffices, by [1, Lemma 2], to show that for every prime ideal $p$ in $R$ of height $\geqslant 2$, we have depth $R_{p} T_{\mathfrak{p}} \geqslant 2$. By [2, Proposition 2.11], $\operatorname{depth}_{R_{\mathfrak{p}}} T_{\mathfrak{p}}=\operatorname{depth}_{T_{\mathfrak{p}}} T_{\mathfrak{p}}$. But depth $T_{\mathfrak{p}} T_{\mathfrak{p}}=$ Krull dim. $T_{\mathfrak{p}}$ (since the Macauley property is preserved by localization) and Krull dim. $T_{p}=$ Krull dim. $R_{\mathfrak{p}}$ (since $T_{\mathfrak{p}}$ is a finite extension of $R_{\mathfrak{p}}$ ). The latter is $\geqslant 2$, by assumption, and so $T$ is $R$-reflexive.

COROLLARY 3.4. Let $A$ be an integrally closed noetherian domain which is Macauley. Let $G$ be a finite group of automorphisms of $A$. Then $A^{G}$ is integrally closed. If $A$ is a finite $A^{G}$-module, then $A$ is $A^{G}$-reflexive, and $A^{G}$ is noetherian. If $o(G)$ is invertible in $A$, then $A^{G}$ is Macauley and is an $A^{G}$-direct summand of $A$.

Proof. Each $A$-automorphism extends to an $F$-automorphism, where $F$ is the quotient field of $\Lambda$. Thus $A^{G}=A \cap F^{G}$, and since $A$ is integrally closed in $F$ it follows immediately that $A^{G}$ is integrally closed in $F^{G}$. If $A$ is a finite $A^{G}$. module, then $A^{G}$ is noetherian, by [4, Theorem 1]. The remaining assertions follow directly from the preceding results in this section.

Note. An example of Nagarajan [11] shows that the hypothesis in Corollary 3.4 that $A$ is a finite $A^{G}$-module, is not superfluous. A regular local ring $A$ and an automorphism $\sigma$ are constructed so that $o(\sigma)=2$ and $A^{\sigma}$ is not noetherian. Hence $A$ is not a finite $A^{\sigma}$-module. On the other hand, the characteristic of $A$ is 2 , so $o(\sigma)$ is not invertible in $A$.

An example of M. Bertin [4] shows that if $o(G)$ is not invertible in $A, A^{G}$ may fail to be Macauley.

From now on $A$ will be a commutative noetherian ring and $\sigma$ an automorphism of $A$ of finite order, say $o(\sigma)=t$. We shall denote the fixed ring under the cyclic group $\langle\sigma\rangle$ by $A^{\sigma}$. Let $\Lambda=A[[X ; \sigma]]$ be the skew power series ring over $A$ in one indeterminate $X$. It consists of all the formal power series $\sum_{i=0}^{\infty} a_{i} X^{i}$, with $X \cdot a=\sigma(a) \cdot X$ for all $a \in A$. By a result of Fields [6], gl. dim. $\Lambda=1+$ gl. $\operatorname{dim} . A$. If $A$ is local with maximal ideal $\mathfrak{m}$, then $\Lambda$ is local with maximal ideal $\mathrm{m} \Lambda+X \Lambda$. If $A$ is a domain, then $\Lambda$ is an order in a division ring. For $\Lambda$ is a noetherian prime ring and thus, by Goldie's thoerem, an order in a simple artin ring $\Sigma$. On the other hand, if $F$ is the quotient field of $A$, then $\Lambda \subset F[[X ; \tau]]$ (where $\tau$ is the extension of $\sigma$ to $F$ ) and so $\Lambda$ is a subring of the division ring 
$F[[X ; \tau]]\left[X^{-1}\right]$. Therefore the simple artin ring $\Sigma$ is a subring of this division ring, and hence is itself a division ring.

A simple calculation shows that the center $R$, of $\Lambda$, is $A^{\sigma}\left[\left[X^{t}\right]\right]$. Again, by Fields' result, gl. $\operatorname{dim} . R=1+\mathrm{gl} . \operatorname{dim} . A^{\sigma}$.

THEOREM 3.5. Let $A$ be a regular local ring, and assume that the A-automorphism $\sigma$ has order $t$ which is invertible in $A$. Assume that $A$ is a finite $A^{\sigma}$ module. Let $\Lambda=A[[X ; \sigma]]$. Then its center $R$ is a noetherian, integrally closed, local Macauley domain. $\Lambda$ is local, and gl. $\operatorname{dim} . \Lambda=\mathrm{Krull} \operatorname{dim} . R$. Furthermore, $\Lambda$ is a finitely generated $R$-reflexive $R$-module, and $R$ is an $R$-direct summand of $\Lambda$.

Proof. As noted above, $R=A^{\sigma}\left[\left[X^{t}\right]\right]$. By Corollary $3.4, A^{\sigma}$ is integrally closed, noetherian, and Macauley. Since $A^{\sigma}=A \cap F^{\tau}, A$ is local, and $F^{\tau}$ is a field, it is easy to see that the set of nonunits in $A^{\sigma}$ is closed under addition, and hence $A^{\sigma}$ is local. All these properties are inherited by $R$, since it is a power series ring over $A^{\sigma}$. By Corollary $3.4, A$ is $A^{\sigma}$-reflexive, and hence $A\left[\left[X^{t}\right]\right]$ is $A^{\sigma}\left[\left[X^{t}\right]\right]$-reflexive. Also, the finiteness of $A$ over $A^{\sigma}$ implies the finiteness of $A\left[\left[X^{t}\right]\right]$ over $A^{\sigma}\left[\left[X^{t}\right]\right]$. Now $A[[X ; \sigma]]$ is free over $A\left[\left[X^{t}\right]\right]$ on the basis $\{1$, $\left.X, \ldots, X^{t-1}\right\}$, and so $A[[X ; \sigma]]$ is finitely generated $A^{\sigma}\left[\left[X^{t}\right]\right]$-reflexive.

$A^{\sigma}$ is an $A^{\sigma}$-summand of $A$ (Corollary 3.4) and hence $R=A^{\sigma}\left[\left[X^{t}\right]\right]$ is an $R$-summand of $A\left[\left[X^{t}\right]\right]$. Since $\Lambda=A[[X ; \sigma]]$ is $A\left[\left[X^{t}\right]\right]$-free, $R$ is an $R$-summand of $\Lambda$.

As we noted in the preliminary remarks, gl. $\operatorname{dim} . \Lambda=1+\mathrm{gl}$. dim. $A$. Now gl. $\operatorname{dim} . A=\operatorname{Krull} \operatorname{dim} . A=\operatorname{Krull} \operatorname{dim} . A^{\sigma}$, and Krull dim. $A^{\sigma}\left[\left[X^{t}\right]\right]=1+$ Krull dim. $A^{\sigma}$. Therefore gl. $\operatorname{dim} . \Lambda=$ Krull dim. $R$.

This theorem suggests the following

Conjecture. The $R$-order $\Lambda$ in Theorem 3.5 is maximal.

REMARK. Since gl. $\operatorname{dim} . R=1+\mathrm{gl} . \operatorname{dim} . A^{\sigma}, R$ is regular local if and only if $A^{\sigma}$ is. Thus, to obtain an example in which $\Lambda$ has global dimension $n+1$ but its center $R$ has infinite global dimension, one need only find a regular local ring $A$ of dimension $n$ and an automorphism $\sigma$ of finite order such that $A^{\sigma}$ is not regular local.

EXAmple 1. Let $k$ be a field of characteristic $\neq 2$, and let $A=k[[Y, Z]]$, $Y$ and $Z$ independent indeterminates. Define $\sigma: A \rightarrow A$ by $\sigma(Y)=-Y$, $\sigma(Z)=-Z$, and $\left.\sigma\right|_{k}=$ identity. Then $o(\sigma)=2$ and $A^{\sigma}=k\left[\left[Y^{2}, Z^{2}, Y Z\right]\right] . A^{\sigma}$ is not factorial since $Y^{2} \cdot Z^{2}=(Y Z)^{2}$, and hence it is not regular, i.e. gl. $\operatorname{dim} . A^{\sigma}=\infty$. Let $\Lambda=A[[X ; \sigma]]$. Its center, $R$, is $A^{\sigma}\left[\left[X^{2}\right]\right]$. Thus gl. $\operatorname{dim} . \Lambda=3$, while gl. $\operatorname{dim} . R=\infty$.

We shall now show that as an $R$-order in the division algebra $\Lambda \otimes_{R} K, \Lambda$ 
is maximal. Since $\Lambda$ is $R$-reflexive, it suffices, by [3, Theorem 1.5], to prove that for every height one prime $\mathfrak{p}$ of $R, \Lambda_{\mathfrak{p}}$ is a maximal $R_{\mathfrak{p}}$-order. Since gl. $\operatorname{dim} . \Lambda=3$, it follows from Corollary 1.3 that gl. $\operatorname{dim} . \Lambda_{p}=1$, i.e. $\Lambda_{p}$ is hereditary. Hence, by [3, Theorem 2.3], $\Lambda_{\mathfrak{p}}$ is maximal if and only if it is quasilocal.

As an $R$-module, $\Lambda$ is generated by $\{1, Y, Z, X, X Y, X Z\}$. Since the height of $\mathfrak{p}$ is one, either $Y^{2} \notin \mathfrak{p}$ or $Z^{2} \notin \mathfrak{p}$. We may assume, with no loss in generality, that $Z^{2} \notin \mathfrak{p}$. Then $Y=\left(Y Z / Z^{2}\right) \cdot Z \in R_{\mathfrak{p}} Z$, and so $\Lambda_{\mathfrak{p}}=R_{\mathfrak{p}}\{1, Z, X, X Z\}$. Since $\left[\Lambda_{\mathfrak{p}} / \mathfrak{p} \Lambda_{\mathfrak{p}}: R_{\mathfrak{p}} / \mathfrak{p} R_{\mathfrak{p}}\right]=\left[\Lambda_{\mathfrak{p}} \otimes_{R_{\mathfrak{p}}} K: K\right]=4,\{1, \bar{Z}, \bar{X}, \overline{X Z}\}$ is a basis of $\Lambda_{\mathfrak{p}} / \mathfrak{p} \Lambda_{\mathfrak{p}}$ over the field $R_{\mathfrak{p}} / \mathfrak{p} R_{\mathfrak{p}} . \bar{X} \bar{Z}=-\bar{Z} \bar{X}$, and $\bar{X}^{2}, \bar{Z}^{2} \in R_{\mathfrak{p}} / \mathfrak{p} R_{\mathfrak{p}}$, with $\bar{Z}^{2} \neq 0$. If $X^{2} \notin \mathfrak{p}$, then $\bar{X}^{2} \neq 0$ and $\Lambda_{\mathfrak{p}} / \mathfrak{p} \Lambda_{\mathfrak{p}}$ is a quaternion algebra over $R_{\mathfrak{p}} / \mathfrak{p} R_{\mathfrak{p}}$ and hence a central simple $R_{\mathfrak{p}} / \mathfrak{p} R_{\mathfrak{p}}$-algebra. Therefore $J\left(\Lambda_{\mathfrak{p}}\right)=\mathfrak{p} \Lambda_{\mathfrak{p}}$ and $\Lambda_{\mathfrak{p}}$ is quasi-local. On the other hand, if $X^{2} \in \mathfrak{p}$, then $X \in J\left(\Lambda_{\mathfrak{p}}\right)$. Now $\Lambda_{\mathfrak{p}} / X \Lambda_{\mathfrak{p}}=R_{\mathfrak{p}} / \mathfrak{p} R_{\mathfrak{p}}[\bar{Z}]$, which is a field. Thus $\Lambda_{\mathfrak{p}}$ is local. So for every height one prime $\mathfrak{p}, \Lambda_{\mathfrak{p}}$ is quasi-local, and therefore $\Lambda$ is maximal.

EXAMPLE 2. Let $\Lambda$ and $R$ be the same as in the preceding example. Let $Q=\left(Y^{2}, Z^{2}, Y Z\right) R$. Then $Q$ is a height two prime of $R$. Gl. $\operatorname{dim} . \Lambda_{Q}=$ Krull dim. $R_{Q}=2$. Clearly the minimal number of generators of $Q R_{Q}$ is 3 , so $R_{Q}$ is not regular. $J\left(\Lambda_{Q}\right) \supset Q \Lambda_{Q} \supset(Y, Z)^{2}$, so $(Y, Z) \subset J\left(\Lambda_{Q}\right)$. Hence $\Lambda_{Q} / J\left(\Lambda_{Q}\right) \simeq k\left(\left(X^{2}\right)\right)$, the quotient field of $k\left[\left[X^{2}\right]\right]$. Thus $\Lambda_{Q}$ is local. Finally, since $R$ is an $R$-summand of $\Lambda, R_{Q}$ is an $R_{Q}$-summand of $\Lambda_{Q}$. We therefore have an example of an order satisfying the hypotheses of Theorem 2.2, whose center is not regular local.

Finally, we note the following example of Jensen and J/ndrup [9], which is similar to, but simpler than, our Example 2. Using a skew power series ring in 2 variables over a field, they construct a left and right noetherian local domain $\Lambda$ of global dimension 2, finitely generated over its center $R$, and $R$ is a complete local domain which is not regular (i.e. which has infinite global dimension).

EXAMPLE 3 (JENSEN AND J $\phi$ NDRUP). Let $\mathbf{C}$ be the field of complex numbers, $\mathbf{R}$ the reals, and let $\sigma$ denote the automorphism of $\mathbf{C}$ given by complex conjugation. Let $\Lambda=\mathrm{C}[[X, Y ; \sigma]]$, the ring of formal power series in $X$ and $Y$ with complex coefficients, with relations $X \cdot Y=Y \cdot X, X \cdot a=\sigma(a) \cdot X$, and $Y \cdot a=$ $\sigma(a) \cdot Y$, for all $a \in \mathbf{C}$. The center $R$ is $\mathbf{R}\left[\left[X^{2}, Y^{2}, X Y\right]\right]$.

In addition to the properties of $\Lambda$ and $R$ mentioned above, $R$ is an $R$-direct summand of $\Lambda$. Hence $\Lambda$ is an example of an order satisfying the hypotheses of Theorem 2.3, whose center has infinite global dimension.

\section{REFERENCES}

1. M. Auslander, Remarks on a theorem of Bourbaki, Nagoya Math. J. 27 (1966), 361-369. MR 33 \#1330. 
2. M. Auslander and D. A. Buchsbaum, Codimension and multiplicity, Ann. of Math. (2) 68 (1958), 625-657. MR 20 \#6414.

3. M. Auslander and O. Goldman, Maximal orders, Trans. Amer. Math. Soc. 97 (1960), 1-24. MR 22 \#8034.

4. M.-J. Bertin, Anneaux d'invariants d'anneaux de polynômes, en caractéristique $p$, C. R. Acad. Sci. Paris Sér. A-B 264 (1967), A653-A656. MR 35 \#6661.

5. D. Eisenbud, Subrings of Artinian and Noetherian rings, Math. Ann. 185 (1970), 247-249. MR 41 \#6885.

6. K. L. Fields, On the global dimension of skew polynomial rings, J. Algebra 13 (1969), 1-4. MR 39 \#6929.

7. M. Harada, Hereditary orders, Trans. Amer. Math. Soc. 107 (1963), 273-290. MR 27 \#1474.

8. M. Hochster and J. A. Eagon, Cohen-Macauley rings, invariant theory, and the generic perfection of determinantal loci, Amer. J. Math. 93 (1971), 1020-1058. MR 46 \#1787.

9. C. U. Jensen and S. J $\phi$ ndrup, Centres and fixed-point rings of Artinian rings, Math. Z. 130 (1973), 189-197. MR 47 \#6769.

10. I. Kaplansky, Commutative rings, Allyn and Bacon, Boston, Mass., 1970. MR 40 $\# 7234$.

11. K. R. Nagarajan, Groups acting on Noetherian rings, Nieuw Arch. Wisk. (3) 16 (1968), 25-29. MR 37 \#5202.

12. M. Nagata, Local rings, Interscience Tracts in Pure and Appl. Math., no. 13, Interscience, New York and London, 1962. MR 27 \#5790.

13. M. Ramras, Maximal orders over regular local rings of dimension two, Trans. Amer. Math. Soc. 142 (1969), 457-479. MR 39 \#6878.

14. - Maximal orders over regular local rings, Trans. Amer. Math. Soc. 155 (1971), 345-352. MR 42 \#7689.

DEPARTMENT OF MATHEMATICS, UNIVERSITY OF MASSACHUSETTS, BOSTON, MASSACHUSETTS 02125 